\title{
Effectiveness of Regional Hyperthermia With Chemotherapy for High-Risk Retroperitoneal and Abdominal Soft-Tissue Sarcoma After Complete Surgical Resection
}

\author{
A Subgroup Analysis of a Randomized Phase-III Multicenter Study
}

\author{
Martin K. Angele, MD, ${ }^{*}$ Markus Albertsmeier, MD, ${ }^{*}$ Niclas J. Prix, MD, ${ }^{*}$ Peter Hohenberger, MD, \\ Sultan Abdel-Rahman, MS, $\dagger$ Nelli Dieterle, $\dagger$ Michael Schmidt, MD, $\S$ Ulrich Mansmann, $\S$ \\ Christiane J. Bruns, MD, $\uparrow$ Rolf D. Issels, MD, $\dagger$ Karl-Walter Jauch, MD, ${ }^{*}$ and Lars H. Lindner, MD $\dagger$
}

\begin{abstract}
Objective: To determine whether regional hyperthermia (RHT) in addition to chemotherapy improves local tumor control after macroscopically complete resection of abdominal or retroperitoneal high-risk sarcomas.

Background: Within the prospectively randomized EORTC 62961 phaseIII trial, RHT and systemic chemotherapy significantly improved local progression-free survival (LPFS) and disease-free survival (DFS) in patients with abdominal and extremity sarcomas. That trial included macroscopically complete and $\mathrm{R} 2$ resections.

Methods: A subgroup analysis of the EORTC trial was performed and longterm survival determined. From 341 patients, 149 (median age 52 years, 18-69) were identified with macroscopic complete resection (R0, R1) of abdominal and retroperitoneal soft-tissue sarcomas (median diameter $10 \mathrm{~cm}, \mathrm{G} 2$ 48.3\%, G3 51.7\%). Seventy-six patients were treated with EIA (etoposide, ifosfamide, doxorubicin) + RHT ( $\geq 5$ cycles: 69.7\%) versus 73 patients receiving EIA alone ( $\geq 5$ cycles: $52.1 \%, P=0.027$ ). LPFS and DFS as well as overall survival were determined.

Results: RHT and systemic chemotherapy significantly improved LPFS (56\% vs $45 \%$ after 5 years, $P=0.044)$ and DFS $(34 \%$ vs $27 \%$ after 5 years, $P=$ $0.040)$. Overall survival was not significantly improved in the RHT group $(57 \%$ vs $55 \%$ after 5 years, $P=0.82$ ). Perioperative morbidity and mortality were not significantly different between groups.

Conclusions: In patients with macroscopically complete tumor resection, RHT in addition to chemotherapy resulted in significantly improved local tumor control and DFS without increasing surgical complications. Within a multimodal therapeutic concept for abdominal and retroperitoneal high-risk sarcomas, RHT is a treatment option beside radical surgery and should be further evaluated in future trials.
\end{abstract}

Keywords: local progression-free survival, multimodal therapy, retroperitoneal sarcoma, surgical resection

(Ann Surg 2014;260:749-756)

From the * Department of General, Visceral, Transplantation, Vascular, and Thoracic Surgery and; †Department of Internal Medicine III, University of Munich-Campus Grosshadern, Munich, Germany; $\ddagger$ Sarcoma Unit ITMInterdisciplinary Tumor Center Mannheim, Mannheim University Medical Center, University of Heidelberg, Mannheim, Germany; §Institute of Medical Informatics, Biometry, and Epidemiology, University of Munich-Campus Großhadern, Munich, Germany; and $\uparrow$ Department of General, Visceral, and Vascular Surgery, University Hospital Magdeburg, University of Magdeburg, Magdeburg, Germany.

M.K.A. and M.A. contributed equally to this work.

Disclosure: This study was supported by Deutsche Krebshilfe (M99/94/Wi II, 70-I702-Wi II), Helmholtz Association (VHVI-140), EORTC 62961, ESHO RHT-95, and NIH P01 CA42745. The authors declare no conflicts of interest. Reprints: Martin K. Angele, MD, Department of General, Visceral, Transplantation,

Vascular, and Thoracic Surgery, University of Munich - Campus Grosshadern,

Ludwig-Maximilians University, Marchioninistr. 15, 81377 Munich, Germany.

E-mail: Martin.Angele@med.uni-muenchen.de.

Copyright (C) 2014 by Lippincott Williams \& Wilkins

ISSN: 0003-4932/14/26005-0749

DOI: $10.1097 /$ SLA.0000000000000978
$\mathrm{R}$ etroperitoneal and abdominal soft-tissue sarcomas are rare tumors with a reported incidence of 2.7 cases per million. ${ }^{1}$ For localized abdominal and retroperitoneal tumors, surgical resection with gross negative margins remains the standard of care. As opposed to extremity sarcoma, mortality from tumors located in the retroperitoneum occurs mainly because of local relapse. Local recurrence is responsible for 3 of 4 tumor-related deaths. ${ }^{2}$ Systematic compartmental resection, that is, removal of the tumor en bloc with a rim of normal tissue, including macroscopically unaffected structures, has been shown to reduce local recurrence rates. ${ }^{3-5}$ These studies indicate that complete removal of the tumor represents the most important prognostic factor in patients with retroperitoneal soft-tissue sarcoma.

Despite gross complete multivisceral resections, small surgical margins may occur in the retroperitoneum in patients with large soft-tissue sarcoma. ${ }^{3,6}$ To improve local tumor control, multimodal therapeutic concepts with radiation and systemic perioperative chemotherapy have been developed in addition to radical surgery. ${ }^{7-9}$ The prospective randomized multicenter trial EORTC (European Organisation for Research and Treatment of Cancer) 62961 indicated that hyperthermia combined with systemic chemotherapy with etoposide, ifosfamide, and doxorubicin (EIA) significantly increased local progression-free survival (LPFS) and disease-free survival (DFS) compared to chemotherapy alone in patients with high-risk sarcoma (diameter $>5 \mathrm{~cm}$, FNCLCC grading 2 or 3 ). ${ }^{10}$ This study, however, included a heterogeneous patient collective with retroperitoneal and extremity soft-tissue sarcomas, as well as different resection status (R0/R1 vs R2 resections). Therefore, it remains unknown whether hyperthermia in addition to chemotherapy compensates for insufficient surgery or is also effective in decreasing local recurrence in patients after macroscopically complete resection of sarcomas. Because from a surgical, anatomical, and prognostic perspective, extremity, abdominal, and retroperitoneal sarcoma represent different entities, this manuscript will focus only on abdominal and retroperitoneal sarcoma. ${ }^{11,12}$ Aim of this study was to evaluate the effect of hyperthermia in the subgroup of patients with $\mathrm{R} 0 / \mathrm{R} 1$ resection of retroperitoneal and abdominal sarcoma on local-recurrence, progression-free, and overall long-term survival. Moreover, the effect of the intensified treatment in the regional hyperthermia (RHT) group on perioperative morbidity and mortality was evaluated.

\section{METHODS}

\section{Trial Design}

The EORTC 62961 trial is a multicenter, randomized phase III trial. It has been registered with ClinicalTrials.gov (NCT 00003052) and has been published previously. ${ }^{10}$ The study protocol was approved by the EORTC, and approval from institutional review boards was obtained at all 9 participating centers. 


\section{Patients}

Between 1997 and 2006, a total number of 341 patients (age 18-70 years) with abdominal and extremity soft-tissue sarcomas were included. Eligible patients had the following risk factors: Fédération Nationale des Centres de Lutte contre le Cancer (FNCLCC) Grade 2 and 3, tumor diameter more than $5 \mathrm{~cm}$. Patients with evidence of distant disease were excluded. Written and informed consent was obtained for all patients.

\section{Total Patient Cohort}

Tumor resection was performed within a multimodal therapeutic approach. Patients were randomly assigned to 2 groups. Patients in group "EIA" $(\mathrm{n}=172)$ received perioperative chemotherapy consisting of etoposide, ifosfamide, and doxorubicin (EIA: etoposide $250 \mathrm{mg} / \mathrm{m}^{2}$ on day 1 and 4 , ifosfamide $6 \mathrm{~g} / \mathrm{m}^{2}$ on days $1-4$, doxorubicin $50 \mathrm{mg} / \mathrm{m}^{2}$ on day 1 every 3 weeks). Patients in group "EIA + RHT" $(n=169)$ received chemotherapy according to the same protocol plus RHT (tumor temperatures of $42^{\circ} \mathrm{C}$ for 60 minutes were given on day 1 and day 4 of each EIA cycle). RHT and thermal mapping were done according to the ESHO guidelines for quality and safety assurance. ${ }^{13}$ The BSD-2000 hyperthermia system (BSD Medical Corporation, Salt Lake City, UT) was used. Blinding was not possible due to the nature of the allocated treatment.

After 4 cycles of induction chemotherapy, tumor response was evaluated according to World Health Organization criteria ${ }^{14}$ and confirmed blinded and independently by board members of the EORTC Soft Tissue and Bone Sarcoma Group. Patients who developed progressive disease after 4 cycles did not receive further systemic therapy according to the EORTC 62961 trial protocol. Patients with stable disease or a positive tumor response continued to receive chemotherapy cycles 5 to 8 .

For radiotherapy, if indicated, a total dose of 50 to 60 Gy was delivered, with daily fractions of 1.8 to $2.0 \mathrm{~Gy}$, administered 4 to 6 weeks after surgery. The recommended doses were adjusted depending on the individual situation and potential radiosensitive tissue within the radiation field.

\section{Subgroup Analysis}

To evaluate the effect of RHT particularly in patients with abdominal and retroperitoneal soft-tissue sarcoma, we performed a subgroup analysis of the earlier described EORTC 62961 trial (Fig. 1). ${ }^{10}$ A total of 192 patients with retroperitoneal and abdominal sarcomas were identified ( $\mathrm{N}=192$, EIA + RHT 96 vs EIA 96). From this

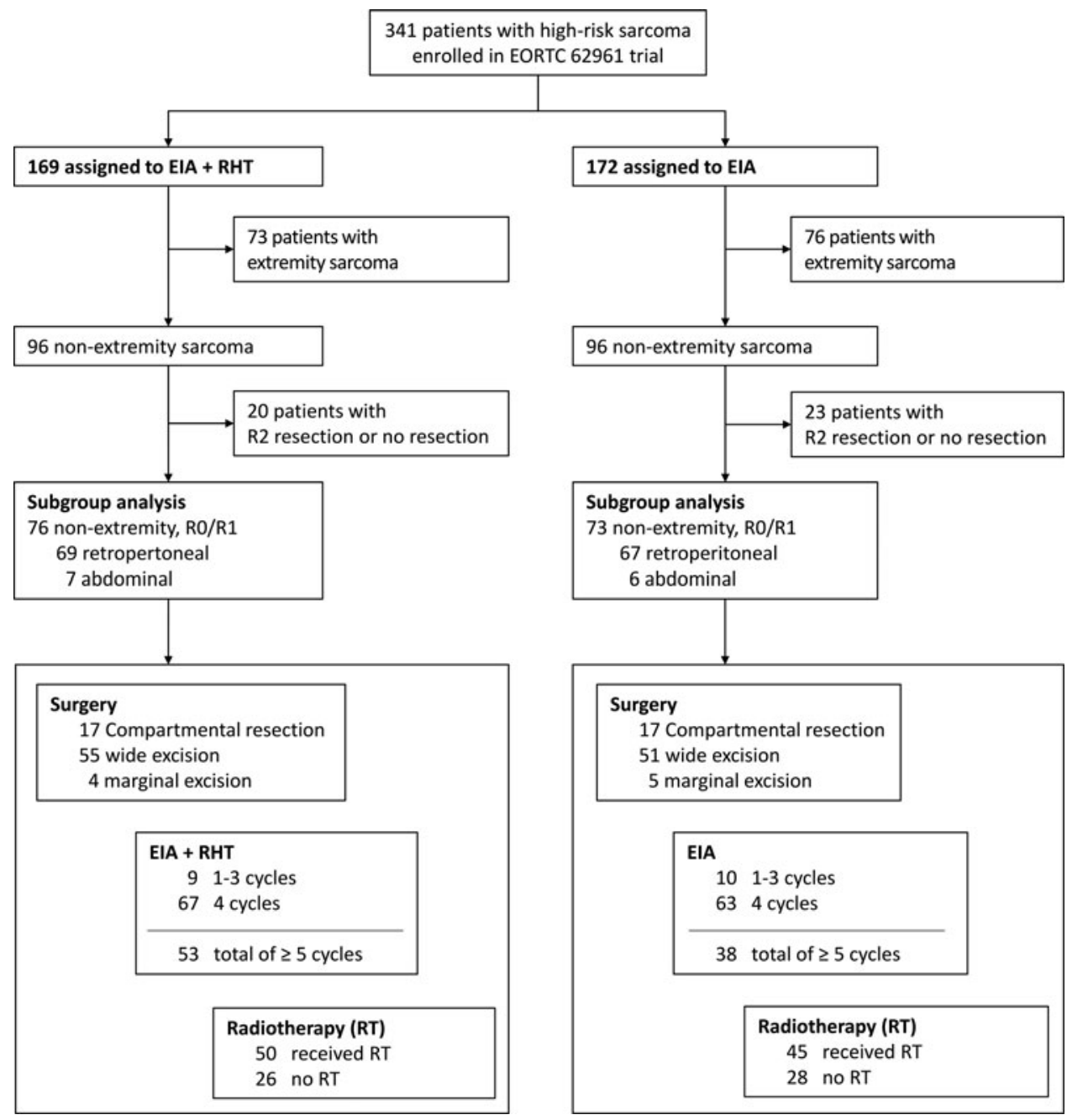

FIGURE 1. Profile of the EORTC 62961 trial and process of subgroup selection. 
cohort, patients $(\mathrm{n}=149,77.6 \%$ ) with macroscopically complete resections (R0, R1) were selected for the present analysis. Resection status was reviewed by an independent pathologist not involved in the study. Patients with intraoperative tumor rupture during operation were considered as $\mathrm{R} 2$ resections.

\section{Primary and Secondary Endpoints}

Patients' recurrence status was documented every 3 months during the first year, every 4 months up to 3 years, every 6 months up to 5 years, and yearly thereafter. Thus, median follow-up was increased from 34 months [interquartile range (IQR): 20-67] in the original publication to 99 months (IQR 20-185) in this article (Fig. 2).

LPFS was chosen as the primary endpoint of this study. It was defined as the time between randomization to confirmed local progression, relapse, or death, whichever occurred first and irrespective of
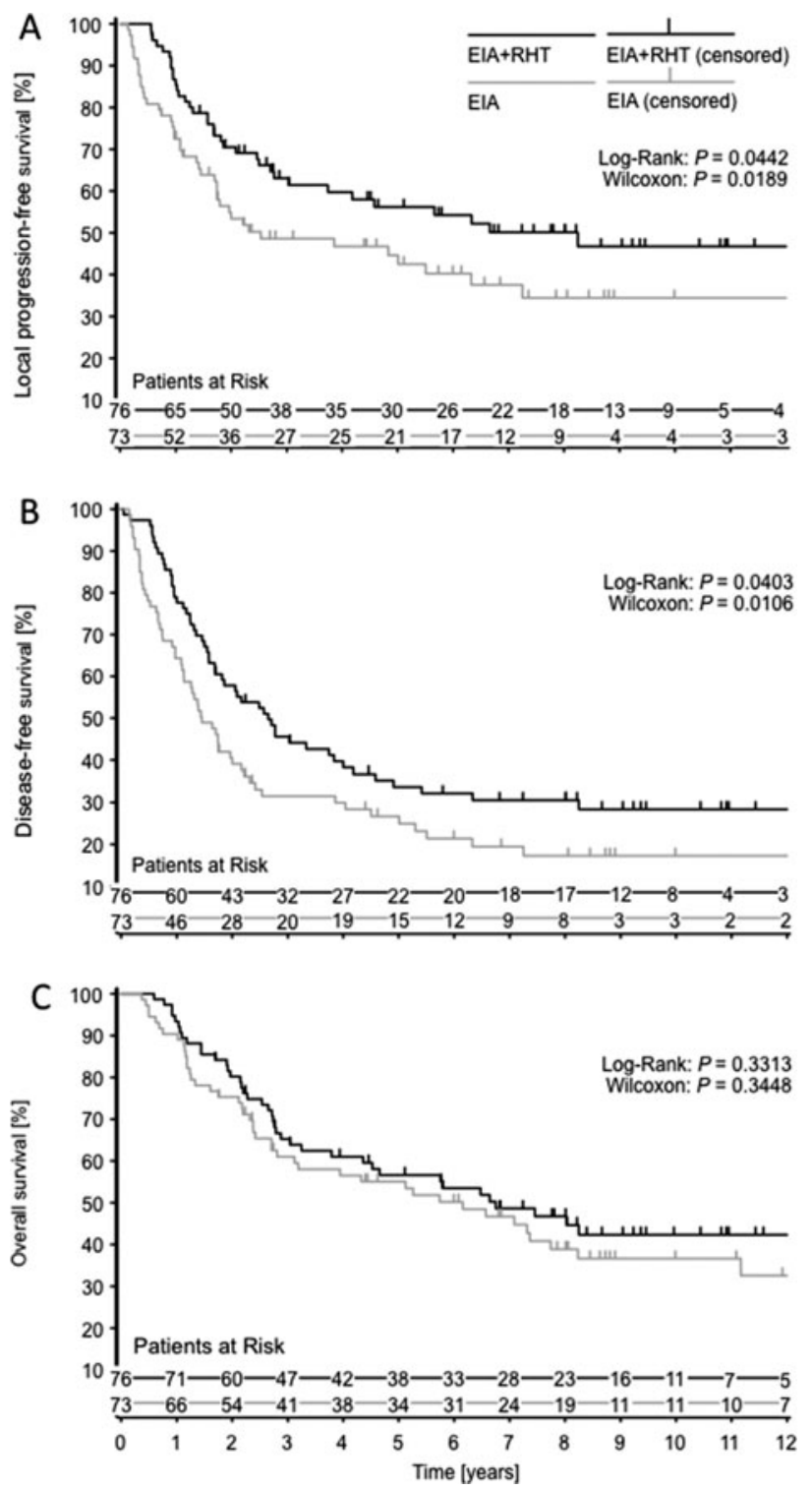

FIGURE 2. Kaplan-Meier estimates of LPFS (A), DFS (B), and OS (C). any occurrence of distant metastases. For patients without confirmed progression, recurrence, metastasis or death, data were censored at the time of the last valid assessment.

Secondary endpoints were DFS, overall survival (OS), tumor response after induction therapy, and perioperative morbidity and mortality. DFS was outlined as the time between randomization and confirmed local failure, distant metastases, or death due to disease or treatment, whichever occurred first. OS was defined as the time between randomization to death of any cause, with survivors being censored at the time of last follow-up. Perioperative mortality was defined as death occurring within 30 days postoperatively. Perioperative morbidity was monitored prospectively including infections, vascular complications, bleeding, neurological complications, etc.

\section{Statistical Analysis}

Sample size was not calculated but determined by the definition of subgroups in this retrospective analysis. Proportions were compared by $\chi^{2}$ tests, and continuous outcomes by Mann-Whitney $U$ tests. Time-to-event data were analyzed by the Kaplan-Meier method. Differences in survival were assessed by stratified log-rank test. A level of significance of 0.05 was applied to all tests. We assessed treatment differences in selected subgroups by stratified Cox regression. All analyses were done using statistical software SAS (version 9.2, SAS Institute, Cary, NC).

\section{RESULTS}

\section{Patient Characteristics}

Median age was 52 years (IQR: 18-69) [53 years, (IQR: 1969) EIA + RTH vs 49 years, (IQR 18-69) EIA, $P=0.33$ ]. Gender distribution was similar in both study groups $(P=0.35)$. Median tumor diameter was $12.0 \mathrm{~cm}$ (IQR: 5-36 cm) in the EIA + RHT group versus $11.0 \mathrm{~cm}$ (IQR: $5-40 \mathrm{~cm})$ in the EIA group $(P=0.41)$. Histopathological findings did not differ between the study groups $(P=0.87)$. Baseline characteristics of the patients included are outlined in Table 1.

A primary tumor was resected in 133 patients [69 $(90.8 \%)$ EIA + RHT vs. 64 (87.7\%) EIA, $P=0.54]$. Locally recurred tumors were treated within the study in 16 cases [7 (9.2\%) EIA + RHT vs 9 (12.3\%) EIA].

\section{Surgical Treatment}

In all patients included, macroscopically complete tumor resection was achieved (R0/R1 resection). As surgical procedure, compartmental resection was performed in $22.8 \%$ (17 EIA + RHT vs 17 EIA, ns) (Table $1, P=0.20$ for all procedures). Most patients $(71.2 \%)$ underwent wide excision as surgical treatment (55 EIA + RHT vs 51 EIA, ns). Marginal excision still classified as R1 resection was accomplished in 6\% (4 EIA + RHT vs 5 EIA, ns).

Morbidity rate in all patients was $19.5 \%$. RHT did not significantly affect postoperative complication rates $(21.0 \%$ EIA + RHT vs $17.9 \%$ EIA, $P=0.62$ ). Specific complications are outlined in Table 2. In addition, median postoperative hospital stay did not differ between the study groups (14 days EIA + RHT vs 12 days EIA). There was no perioperative mortality.

\section{Chemotherapy \pm RHT}

In the EIA + RHT group, 67 patients $(88.2 \%)$ received all 4 cycles of induction chemotherapy, whereas in the EIA group, 63 patients $(86.3 \%)$ received all 4 cycles. As for the total number of chemotherapy cycles, 5 or more systemic cycles were applied in 53 patients $(69.7 \%)$ in the EIA + RHT versus 38 patients $(52.1 \%)$ in the EIA group $(P=0.027)$. 
TABLE 1. Patient Characteristics and Demographics

\begin{tabular}{|c|c|c|c|}
\hline & EIA + RHT $(n=76)$ & $\operatorname{EIA}(n=73)$ & $\boldsymbol{P}$ \\
\hline Age, median (range), yr & $53.0(19.0-69.0)$ & $49.0(18.0-69.0)$ & 0.3261 \\
\hline Sex & & & 0.3520 \\
\hline Male & $39(51.3 \%)$ & $43(58.9 \%)$ & \\
\hline Female & $37(48.7 \%)$ & $30(41.1 \%)$ & \\
\hline Presentation of tumor & & & 0.5388 \\
\hline Primary & $69(90.8 \%)$ & $64(87.7 \%)$ & \\
\hline Recurrent & $7(9.2 \%)$ & $9(12.3 \%)$ & \\
\hline Size of tumor, median (range) & $12.0(5.0-36.0)$ & $11.0(5.0-40.0)$ & 0.4120 \\
\hline Pathology & & & 0.8730 \\
\hline Liposarcoma & $16(21.1 \%)$ & $13(17.8 \%)$ & \\
\hline Leiomyosarcoma & $18(23.7 \%)$ & $16(21.9 \%)$ & \\
\hline Fibrosarcoma & $7(9.2 \%)$ & $10(13.7 \%)$ & \\
\hline Malignant fibrous histiocytoma & $2(2.6 \%)$ & $1(1.4 \%)$ & \\
\hline Other sarcomas & $33(43.4 \%)$ & $33(45.2 \%)$ & \\
\hline Type of surgery & & & 0.2017 \\
\hline Compartmental resection & $17(22.4 \%)$ & $17(23.4 \%)$ & \\
\hline Wide excision & $55(72.4 \%)$ & $51(69.9 \%)$ & \\
\hline Marginal excision & $4(5.3 \%)$ & $5(6.8 \%)$ & \\
\hline $\begin{array}{l}\text { Days after surgery until discharge, } \\
\text { median (range) }\end{array}$ & $14(6-49)$ & $12(4-76)$ & 0.3521 \\
\hline
\end{tabular}

TABLE 2. Perioperative Morbidity and Mortality

\begin{tabular}{lccc}
\hline Complications & EIA + RHT $(\mathbf{n = 7 6 )}$ & EIA $(\mathbf{n}=\mathbf{7 3})$ & P \\
\hline None & $60(78.9 \%)$ & $60(82.2 \%)$ & 0.617 \\
Morbidity & $16(21.0 \%)$ & $13(17.9 \%)$ & \\
Superficial wound infection & 4 & 2 & \\
Deep infection & 3 & 4 & \\
Bleeding & 6 & 1 & \\
Neurological & 4 & 3 & \\
Skin necrosis & 4 & 8 & 1.000 \\
Other & 11 & 0 & \\
Mortality & 0 & 2 & \\
\hline
\end{tabular}

Response to induction therapy was determined according to World Health Organization criteria in 94 patients (EIA + RHT 49 patients vs EIA 45 patients, ns) after 4 cycles of neoadjuvant treatment. Response rates were significantly higher in the EIA + RHT group than in the EIA group (34.7\% vs $15.6 \%, P=0.034)$. Early progression occurred in 10 patients $(22.2 \%)$ treated only with EIA, whereas no patient in the combined treatment group had early progression $(P<0.001)$. In 55 patients, systemic therapy was applied adjuvantly. Thus, no evidence of disease was present at randomization in those patients.

\section{Radiation Therapy}

Radiation therapy was applied in 95 of 149 patients. The distribution was similar in both study groups [50 patients $(65.8 \%)$ EIA + RHT vs 45 patients (61.6\%) EIA, $P=0.54]$.

\section{LPFS, DFS, and OS}

In patients with macroscopically complete tumor resection, RHT resulted in significantly improved LPFS [hazards ratio (HR) $=$ 0.63 , 95\% CI: $0.40-0.99 ; P=0.044]$. Three-year LPFS was 63 months (EIA + RHT) versus 49 months (EIA only) (Table 3 ).

For the combined treatment group versus the EIA only group the relative hazard for DFS was 0.72 (95\% CI: $0.49-1.05 ; P=0.040$ Log-rank test), with a median duration of 2.7 years versus 1.5 years.

Within the follow-up period of 8.2 years, 84 patients died ( 40 in the combined treatment group vs. 44 in the EIA only group). OS did not differ significantly between the groups $(\mathrm{HR}=0.82,95 \% \mathrm{CI}$ : $0.53-1.26 ; P=0.59)$.

\section{DISCUSSION}

The most common site of first recurrence after surgery for retroperitoneal sarcoma is local relapse. ${ }^{2,15}$ Local recurrence rates of more than $80 \%$ have been reported despite complete surgical resection of high-risk soft-tissue sarcomas, ${ }^{16}$ underlining the need for novel multimodal therapeutic strategies. In this respect, our previously published randomized phase III trial showed that RHT enhances the efficacy of chemotherapy (EIA) in the treatment of high-risk softtissue sarcoma. ${ }^{10}$ Patients treated with RHT and EIA had increased LPFS and DFS compared to patients treated with EIA alone. This study, however, included patients with different tumor locations and resection status. Thus, it remained unknown whether RHT in addition to systemic chemotherapy improves LPFS in a homogeneous patient collective with macroscopically complete resection of abdominal or retroperitoneal soft-tissue sarcoma. Because macroscopically incomplete resections are an independent predictor of unfavorable outcome, ${ }^{6,16,17}$ RHT may have improved LPFS due to the inclusion of surgically inadequate resections. ${ }^{10}$

The present subgroup analysis focuses on patients with R0/R1 resection status, excluding macroscopically incomplete resections. Our results indicate that addition of RHT to systemic perioperative chemotherapy improved local tumor control and DFS as compared to chemotherapy alone also in patients with macroscopically complete 


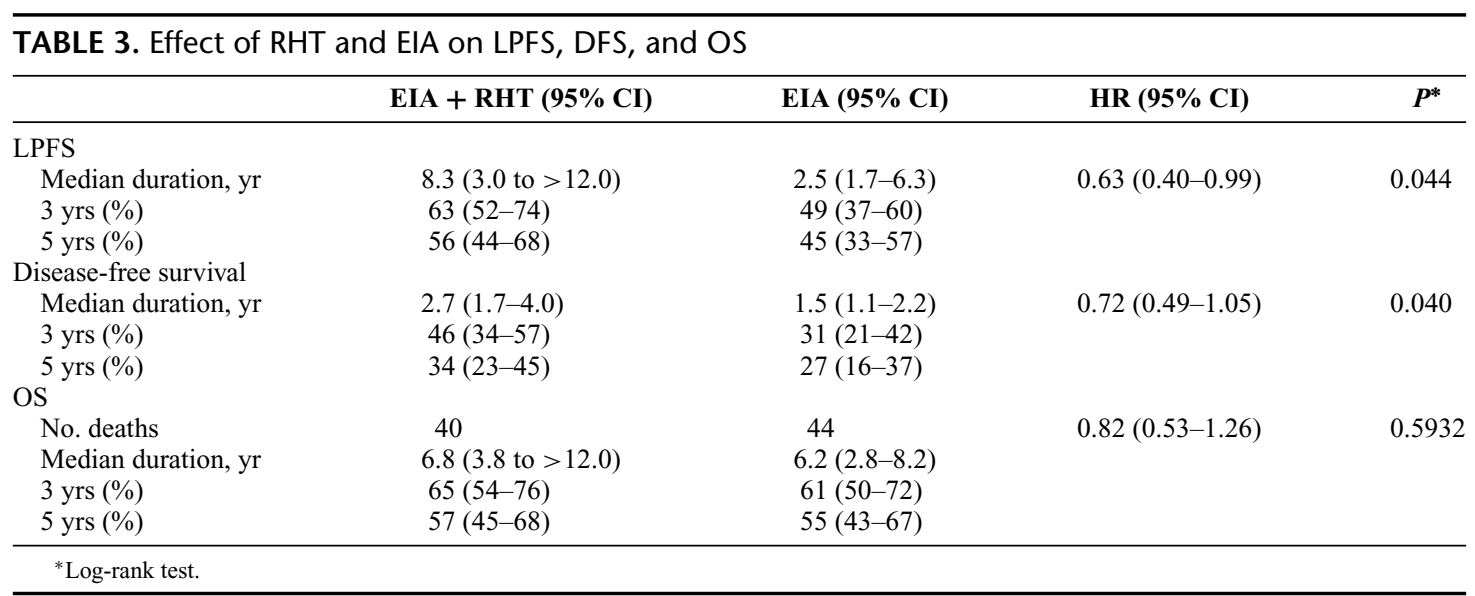

resections. Three-year LPFS was 63 months with RHT as opposed to 49 months in the chemotherapy-only group. There was no effect of RHT on OS. When interpreting these results, it has to be considered that the study represents a subgroup analysis of a prospectively randomized multicenter trial. The lack of statistically significant effects of RHT on OS may be due to the small sample size, which has not been calculated for this subgroup in the initial study design. Moreover, patients who developed progressive disease after 4 cycles of EIA were allowed to receive RHT with cycles 5 to 8 which may have influenced OS.

The tumor response rate after 4 cycles of systemic therapy with EIA + RHT was more than twice as high $(34.7 \%$ vs $15.6 \%$, $P=0.0034)$ when compared to chemotherapy alone. This reflects the effectiveness of the combined treatment approach for local tumor control. ${ }^{10,18,19}$ In particular, no patient in the EIA + RHT group showed early progression, whereas 10 patients $(22.2 \%)$ in the chemotherapy-only group had early progression $(P<0.001)$. As a consequence of reduced early progression, more patients continued to receive systemic therapy cycles 5 to 8 in the EIA + RHT arm.

In the EIA + RHT group, liposarcoma as a histological subtype was slightly more frequent and there were a few more primary tumors (vs recurrent tumors) and wide excisions (vs local tumor resections), although none of these differences was statistically significant. On the contrary, tumors in patients treated only with EIA were smaller by $1 \mathrm{~cm}$. In summary, factors favoring a positive outcome were equally distributed between groups and it seems unlikely that these small differences in patient characteristics are responsible for the observed benefit of RHT on LPFS and DFS. The significantly increased response rate in RHT-treated patients seems to be of greater importance.

Complete surgical tumor resection is the standard of care in retroperitoneal soft tissue sarcomas. Recently, a more aggressive surgical approach with en bloc resection of the tumor together with a rim of normal tissue including uninvolved organs has been introduced in an attempt to further minimize the risk of microscopically positive margins and local relapse. Five-year local recurrence rates of less than $25 \%$ have been reported after systematic compartmental resections compared to $44 \%$ in the RHT group of this study. ${ }^{3-5}$ In those studies, however, more than one third of patients had well-differentiated sarcomas, whereas patients with G1 tumors were specifically excluded in our study protocol. High tumor grade $(\mathrm{G} 2 / \mathrm{G} 3)$ has been shown to negatively influence DFS and OS more than any other tumor-associated risk factor (size, histological subtype, location). ${ }^{20}$ Given that well-differentiated sarcomas are associated with significantly lower recurrence rates and better long-term survival, ${ }^{4,17,20}$ the unequal distribution of tumor grading precludes a comparison of local and distant recurrence-free survival rates.

In this study, less than $25 \%$ of patients were treated with compartmental resection, which was established as a standard procedure after EORTC 62961 started recruiting patients. Because of the small number of patients, further studies are required to evaluate whether RHT in addition to systemic chemotherapy improves the promising results of systematic compartmental resection. Furthermore, extensive multivisceral resections are not feasible in some patients due to accompanying diseases or tumor location. Although previous data presented by Perez et $\mathrm{al}^{17}$ suggest that these patients benefit even from incomplete tumor resections, RHT may improve local tumor control also after $\mathrm{R} 2$ resection of retroperitoneal soft tissue sarcoma.

This study included only high-risk G2/G3 retroperitoneal sarcomas. The rather favorable prognosis of G1 tumors does not justify the use of chemotherapy because of the associated risks of toxicity. Whether radiotherapy improves local tumor control in patients with differentiated sarcomas after radical surgery is being addressed in the ongoing EORTC trial (NCT01344018). We propose the use of EIA and RHT for patients with G2/G3 retroperitoneal sarcomas as part of a differentiated approach tailored to the risk of an individual patient.

The importance of RHT in addition to chemotherapy is emphasized by a recently published study by Woll et al. ${ }^{21}$ This prospective randomized trial failed to demonstrate reduced LPFS for patients receiving adjuvant chemotherapy (doxorubicin, ifosfamide) after macroscopically complete resection of high-risk soft-tissue sarcomas. ${ }^{21}$ To what extent the difference in the chemotherapy protocol and the inclusion of mainly extremity sarcomas contributes to the negative results in that study remains to be determined. In this respect, a retrospective analysis revealed significantly improved DFS in 262 patients with grade 3 sarcoma treated with adjuvant chemotherapy. $^{22}$

Perioperative radiotherapy was applied in $65.8 \%$ of patients in the EIA + RHT group and in $61.6 \%$ of EIA only patients $(P=0.54)$. In a retrospective cohort study based on the SEER (Surveillance, Epidemiology, and End Results) database involving 1901 patients with retroperitoneal sarcoma, perioperative radiotherapy increased LPFS compared to surgery alone. ${ }^{23}$ Systematic perioperative radiotherapy has been shown to be feasible in patients with retroperitoneal sarcomas. ${ }^{24}$ Thus, increasing the number of patients receiving radiotherapy within our multimodal treatment concept may improve local tumor control. The benefit of radiotherapy is currently being evaluated in an ongoing EORTC phase III trial (NCT01344018).

Intensifying chemotherapy via RHT did not increase perioperative complication rates. In this respect, comparable morbidity rates 
between $18 \%$ and $26 \%$ have recently been reported after resection of retroperitoneal sarcomas without neoadjuvant therapy. ${ }^{4,25,26}$ In addition, this study documented all complications including neurological and cardiovascular morbidity whereas most surgical studies reporting lower morbidity rates focused on major complications requiring surgical reintervention. ${ }^{27}$ Most importantly, this trial prospectively recorded all complications whereas previous studies were retrospective and might, therefore, by the nature of their design, have missed some of the complications. Finally, the higher number of completed systemic therapy cycles in the RHT group demonstrates that this novel concept is well-tolerated during systemic therapy and perioperatively. These findings emphasize that improved local tumor control in the RHT group was not achieved at the expense of unacceptable treatment-associated morbidity and mortality.

Contributing centers represented high-volume institutions treating more than 30 soft-tissue sarcomas per year. In particular, high-risk soft tissue sarcomas seem to profit from treatment at highvolume centers. ${ }^{3,4,28}$ This effect of center size has to be considered when interpreting the results of the present trial for the general patient population. Also, the fact that this subgroup analysis was not initially planned may be a source of statistical bias and reflects a limitation of this article.

The previously published EORTC 62961 trial failed to demonstrate improved OS for the whole patient group. ${ }^{10}$ Extending the observation time and focusing on a more homogenous subgroup in the present analysis did not eliminate the discrepancy between significantly improved LPFS/DFS and statistically unaffected OS. Furthermore, $70 \%$ of patients completed more than 4 cycles of systemic therapy. Whether increasing the number of patients completing the entire study protocol or individualizing the use of RHT based on pretherapeutically identified predictive factors will result in improved OS remains to be determined. To date, such predictive factors have not yet been defined.

\section{CONCLUSION}

In summary, addition of RHT to chemotherapy resulted in significantly improved LPFS and DFS after macroscopically complete resection of abdominal and retroperitoneal sarcomas. Intensification of the local effects of perioperative chemotherapy via RHT did not significantly increase surgical complication rates. Whether more aggressive surgical strategies with systematic compartmental resections will further improve local tumor control for patients treated with RHT and chemotherapy remains to be elucidated in future studies.

\section{REFERENCES}

1. Porter GA, Baxter NN, Pisters PW. Retroperitoneal sarcoma: a populationbased analysis of epidemiology, surgery, and radiotherapy. Cancer. 2006;106:1610-1616

2. Stojadinovic A, Yeh A, Brennan MF. Completely resected recurrent soft tissue sarcoma: primary anatomic site governs outcomes. J Am Coll Surg. 2002;194:436-447.

3. Bonvalot S, Rivoire M, Castaing M, et al. Primary retroperitoneal sarcomas: a multivariate analysis of surgical factors associated with local control. J Clin Oncol. 2009;27:31-37.

4. Bonvalot S, Miceli R, Berselli M, et al. Aggressive surgery in retroperitoneal soft tissue sarcoma carried out at high-volume centers is safe and is associated with improved local control. Ann Surg Oncol. 2010;17:1507-1514.

5. Gronchi A, Bonvalot S, Le Cesne A, et al. Resection of uninvolved adjacent organs can be part of surgery for retroperitoneal soft tissue sarcoma. J Clin Oncol. 2009;27:2106-2107; author reply 2107-2108.

6. Singer S, Antonescu CR, Riedel E, et al. Histologic subtype and margin of resection predict pattern of recurrence and survival for retroperitoneal liposarcoma. Ann Surg. 2003;238:358-370; discussion 370-371.

7. Casali PG, Picci P. Adjuvant chemotherapy for soft tissue sarcoma. Curr Opin Oncol. 2005; 17:361-365.
8. Kraybill WG, Harris J, Spiro IJ, et al. Phase II study of neoadjuvant chemotherapy and radiation therapy in the management of high-risk, high-grade, soft tissue sarcomas of the extremities and body wall: Radiation Therapy Oncology Group Trial 9514. J Clin Oncol. 2006;24:619-625.

9. Gortzak E, Azzarelli A, Buesa J, et al. A randomised phase II study on neoadjuvant chemotherapy for "high-risk" adult soft-tissue sarcoma. Eur J Cancer. 2001;37:1096-1103.

10. Issels RD, Lindner LH, Verweij J, et al. Neo-adjuvant chemotherapy alone or with regional hyperthermia for localised high-risk soft-tissue sarcoma: randomised phase 3 multicentre study. Lancet Oncol. 2010;11:561-570.

11. Gutierrez JC, Perez EA, Franceschi D, et al. Outcomes for soft-tissue sarcoma in 8249 cases from a large state cancer registry. J Surg Res. 2007;141:105-114.

12. Colombo C, Randall RL, Andtbacka RH, et al. Surgery in soft tissue sarcoma: more conservative in extremities, more extended in the retroperitoneum. Expert Rev Anticancer Ther. 2012;12:1079-1087.

13. Lagendijk JJ, Van Rhoon GC, Hornsleth SN, et al. ESHO quality assurance guidelines for regional hyperthermia. Int J Hyperthermia. 1998;14:125-133.

14. Miller AB, Hoogstraten B, Staquet M, et al. Reporting results of cancer treatment. Cancer. 1981;47:207-214.

15. Raut CP, Pisters PW. Retroperitoneal sarcomas: combined-modality treatment approaches. J Surg Oncol. 2006;94:81-87.

16. Keung EZ, Hornick JL, Bertagnolli MM, et al. Predictors of outcomes in patients with primary retroperitoneal dedifferentiated liposarcoma undergoing surgery. J Am Coll Surg. 2014;218:206-217.

17. Perez EA, Gutierrez JC, Moffat FL, Jr, et al. Retroperitoneal and truncal sarcomas: prognosis depends upon type not location. Ann Surg Oncol. 2007; $14: 1114-1122$

18. Wendtner C, Abdel-Rahman S, Baumert J, et al. Treatment of primary, recurren or inadequately resected high-risk soft-tissue sarcomas (STS) of adults: results of a phase II pilot study (RHT-95) of neoadjuvant chemotherapy combined with regional hyperthermia. Eur J Cancer. 2001;37:1609-1616.

19. Donahue TR, Kattan MW, Nelson SD, et al. Evaluation of neoadjuvant therapy and histopathologic response in primary, high-grade retroperitoneal sarcomas using the sarcoma nomogram. Cancer. 2010;116:3883-3891.

20. Gronchi A, Miceli R, Shurell E, et al. Outcome prediction in primary resected retroperitoneal soft tissue sarcoma: histology-specific overall survival and disease-free survival nomograms built on major sarcoma center data sets. J Clin Oncol. 2013;31:1649-1655.

21. Woll PJ, Reichardt P, Le Cesne A, et al. Adjuvant chemotherapy with doxorubicin, ifosfamide, and lenograstim for resected soft-tissue sarcoma (EORTC 62931): a multicentre randomised controlled trial. Lancet Oncol. 2012;13:1045-1054.

22. Italiano A, Delva F, Mathoulin-Pelissier S, et al. Effect of adjuvant chemotherapy on survival in FNCLCC grade 3 soft tissue sarcomas: a multivariate analysis of the French Sarcoma Group Database. Ann Oncol. 2010;21:2436 2441.

23. Zhou Z, McDade TP, Simons JP, et al. Surgery and radiotherapy for retroperitoneal and abdominal sarcoma: Both necessary and sufficient. Arch Surg. 2010;145:426-431.

24. Gronchi A, De Paoli A, Dani C, et al. Preoperative chemo-radiation therapy for localised retroperitoneal sarcoma: a phase I-II study from the Italian Sarcoma Group. Eur J Cancer. 2014;50:784-792.

25. Lehnert T, Cardona S, Hinz U, et al. Primary and locally recurrent retroperitoneal soft-tissue sarcoma: Local control and survival. Eur J Surg Oncol. 2009;35:986-993

26. Tseng WH, Martinez SR, Tamurian RM, et al. Contiguous organ resection is safe in patients with retroperitoneal sarcoma: An ACS-NSQIP analysis. J Sur Oncol. 2011;103:390-394.

27. Hassan I, Park SZ, Donohue JH, et al. Operative management of primary retroperitoneal sarcomas: a reappraisal of an institutional experience. Ann Surg. 2004;239:244-250.

28. Gutierrez JC, Perez EA, Moffat FL, et al. Should soft tissue sarcomas be treated at high-volume centers? An analysis of 4205 patients. Ann Surg. 2007;245:952958

\section{DISCUSSANTS}

\section{P. Gertsch (Zurich, Switzerland):}

I thank the association for the privilege of commenting on this interesting and well-presented paper.

The Munich group has shown a persistent interest in introducing hyperthermia for the treatment of malignant tumors using a sophisticated technique developed more than 20 years ago. 
After obtaining results from early retrospective analyses and phase 2 studies, they have completed a multicentric, phase 3 study on the treatment of high-risk soft tissue sarcoma of the abdomen and its extremities, which was published in 2010 in Lancet Oncology. The actual study is presented as a subgroup analysis focused on patients with abdominal sarcomas who were observed over a longer period of time than in the original study. In spite of this longer observation time, it can be considered to be a post hoc subgroup analysis.

To fully understand this study, one has to refer back to the initial report published in 2010. In this randomized controlled trial, LPFS was chosen as the primary endpoint, while an intention-totreat analysis was performed. The randomization was done before the induction therapy, and of course, before surgical treatment. In the subgroup analysis, presented here, patients who had an R2 resection were excluded from the analysis, in addition to those who had R0 or R1 resections, after having sustained an intraoperative rupture of the tumor. I suspect that excluding these patients, after they underwent randomization and surgery, from the selected subgroup can be considered a "protocol violation" in a prospective randomized study, adopting an intention-to-treat analysis.

Despite longer follow-ups, the detected benefits of LPFS and DFS, in the initial study and in the subgroup analysis, were similar, showing a significant advantage for tumors treated with hyperthermia. After 5 years, the differences between LPFS and DFS were $11 \%$ and $7 \%$, respectively. In the absence of the benefit of long-term survival, I have doubts that a small, although significant, advantage has any clinical importance. As such, I have the following question: Did you perform a quality-of-life assessment after administering the treatment? In addition, could you give us information on the cost of the apparatus used for RHT, including the cost per patient undergoing this treatment?

The use of hyperthermia in its various forms is not new. Intraperitoneal hyperthermia, used in the treatment of peritoneal carcinomatosis, has been revealed to have limitations in its application. For instance, though they enhance tumor destruction, temperatures exceeding $43^{\circ} \mathrm{C}$ carry a risk of intestinal damage and perforation. In addition, the technique you have used is more sophisticated and many of us are not familiar with it. How did you assess the limits of the area of treatment as well as the temperatures reached within the tumor and at its margins?

Your description of the complications does not take into account their therapeutic consequences; for example, bleeding was more frequent in patients treated with a combination of chemotherapy and hyperthermia. Did these patients require transfusions, interventional radiology, or surgery? What were the respective causes and consequences of deep infection in both groups and how were these treated?

In the original published study, with respect to abdominal and extremity sarcomas, 2 deaths were attributed to treatment in the hyperthermia group, whereas only 1 was attributed to the chemotherapy group. Is it correct to assume that mortality was limited to extremity sarcomas? Or, did it occur in the patients who you excluded from the analysis following randomization? Did you analyze mortality as you do in the hospital: 30-day or 90-day mortality in your study?

Finally, I would like you to discuss your conclusions in a bit more detail. How can we translate these results to a real-life scenario? If neoadjuvant treatment with hyperthermia is efficient in R0 and R1 unruptured tumor resections, how do we detect these patients preoperatively? It must be said that including $\mathrm{R} 2$ resections in your study would have respected the initial protocol and given more transparent conclusions.

I thank you for having given me your manuscript. I enjoyed your presentation, which provided interesting observations for plan- ning further trials. Innovative ideas for the treatment of these uncommon and aggressive tumors are welcome and deserve to be known.

\section{Response From M. K. Angele (Munich, Germany):}

Thank you very much for these kind comments and good questions. With respect to the quality of life, no such analysis was performed in the patients. A quality-of-life analysis was not a standard procedure at the time we initiated the study. Nonetheless, the higher number of patients, who completed 5 or more cycles of chemotherapy plus hyperthermia, is a good indication of their tolerance to chemotherapy in the RHT arm of the study. It seems that a higher response rate and absence of tumor progression motivates the patients to continue therapy, increasing their quality of life.

The approximate cost for setting up a hyperthermia unit amounts to 2,000,000 €. The additional operating expenses for hyperthermia within 1 chemotherapy cycle are approximately $8000 €$. These costs are covered by the patient's health insurance, in addition to the reimbursement for chemotherapy.

The extent of the hyperthermia area is dependent on the computed tomographic scan or magnetic resonance imaging, which focus on the tumor and its surrounding tissue in the preoperative setting or the area of tumor resection in the postoperative setting. Temperature in the treatment field is measured by invasively inserting temperature probes, which enable us to estimate temperature distribution within the tumor and its margins as well as in the surrounding tissue. This approach assures an adequate temperature distribution within the treatment field.

We agree that the number of bleeding complications was increased in the hyperthermia group, although this increase was not significant. Only one patient had to be reoperated because of a bleeding complication, but this was surgically controlled. We doubt that this case of bleeding was related to the addition of hyperthermia to chemotherapy. Interventional radiology was not required in any of the patients with a bleeding complication.

In 2 patients of each treatment arm, deep infections had to be surgically treated. None of these patients required more than 1 surgical reoperation. The other 3 patients were treated interventionally with computed tomography-guided drainages.

To determine the surgery-associated death rate, we analyzed the 30-day mortality rate. Within our patient collective, no patient died within 30 days. Mortality reported in the original publication was associated with chemotherapy-related complications.

The aim of this study was to verify whether patients with macroscopically complete tumor resection profit from both hyperthermia and chemotherapy combined. Patients with R2 resections were, therefore, excluded from the subgroup analysis. The results demonstrate that hyperthermia does not solely compensate for insufficient surgery but has an additional benefit in patients with gross complete tumor resections. The analysis was not intended to identify patients who may particularly profit from hyperthermia.

\section{DISCUSSANTS}

\section{H. Friess (Munich, Germany):}

Thank you for presenting this important trial on visceral soft tissue sarcomas. I have 2 questions. The first question is the following: how was your management in both groups after a sarcoma recurrence was detected? Was treatment comparable in both patient groups?

Second, what is your present recommendation to patients with a visceral soft tissue sarcoma, based on the results of your study? With regard to LPFS and DFS, RHT seems to offer a significant benefit to patients. However, in the end, they do not live longer, since OS is not influenced. Therefore, do you still recommend RHT to patients 
with visceral soft tissue sarcoma, even though it will not prolong their survival?

\section{Response From M. K. Angele (Munich, Germany):}

Thank you, Professor Friess, for your questions. To answer your first question about the management of recurrence, the management of the patients was similar in both groups. In cases of local recurrence, complete surgical resection was intended within a multimodal treatment concept. The treatment of recurrent diseases, however, was outside the study protocol.
Although OS was not affected by the treatment, the protocol used in this study and at our institution is to recommend patients with high-risk abdominal sarcomas to undergo hyperthermia and chemotherapy perioperatively, in addition to radical surgical treatment. This suggestion is based on the fact that this may significantly improve LPFS, which is associated with less treatment requirements in the hyperthermia group. We, therefore, assume that the decreased local recurrence rate results in an improved quality of life. Moreover, OS, although not significantly, was slightly improved in the RHT group. 